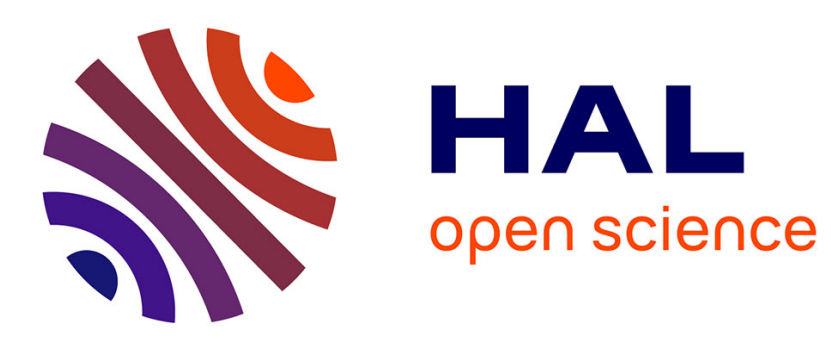

\title{
On the Inertial Single Phase Flow in 2D Model Porous Media: Role of Microscopic Structural Disorder
}

\author{
Yibiao Wang, Azita Ahmadi, Didier Lasseux
}

\section{To cite this version:}

Yibiao Wang, Azita Ahmadi, Didier Lasseux. On the Inertial Single Phase Flow in 2D Model Porous Media: Role of Microscopic Structural Disorder. Transport in Porous Media, 2019, 128 (1), pp.201220. 10.1007/s11242-019-01241-x . hal-02180936

\section{HAL Id: hal-02180936 https://hal.science/hal-02180936}

Submitted on 11 Jul 2019

HAL is a multi-disciplinary open access archive for the deposit and dissemination of scientific research documents, whether they are published or not. The documents may come from teaching and research institutions in France or abroad, or from public or private research centers.
L'archive ouverte pluridisciplinaire HAL, est destinée au dépôt et à la diffusion de documents scientifiques de niveau recherche, publiés ou non, émanant des établissements d'enseignement et de recherche français ou étrangers, des laboratoires publics ou privés. 


\title{
On the Inertial Single Phase Flow in 2D Model Porous Media: Role of Microscopic Structural Disorder
}

\author{
Yibiao Wang ${ }^{1} \cdot$ Azita Ahmadi $^{1} \cdot$ Didier Lasseux $^{2}$ (D)
}

\begin{abstract}
In this work, single-phase incompressible laminar flow in $2 \mathrm{D}$ model porous media is studied and the influence of microscopic structural disorder on the flow is thoroughly investigated. Emphasis is laid upon the onset of the deviation from Darcy's law and the identification of different inertia regimes observed before the flow becomes unsteady. For this purpose, six globally disordered pore structures were generated and the values of the critical Reynolds number at which the flow becomes unsteady corresponding to the first Hopf bifurcation were determined. Numerical simulations of steady laminar single-phase flow were then carried out to investigate the effects of the microstructures on the inertial correction to Darcy's law. Different flow regimes, namely weak inertia, strong inertia and the regime beyond strong inertia, are identified. Comparisons are made with results presented in the literature which were restricted to ordered and locally disordered structures. The critical Reynolds number decreases and inertia intensity increases as more disorder is introduced into the pore structure. Results on flow inertia widely extend some previous studies on the subject and show that it is mainly influenced by the shape of the obstacles (either circular or square), slightly affected by the inclination of the square cylinders and hardly disturbed by the size distribution of the obstacles.
\end{abstract}

Keywords Inertial one-phase flow $\cdot$ Non-Darcy flow $\cdot$ Flow regimes $\cdot$ Darcy-Forchheimer

\section{Introduction}

Inertial flow in porous media corresponds to flow regimes where the Stokes or creeping model is not valid at the microscopic scale so that Darcy's law is not applicable at the macroscopic scale. Such flows are encountered in many practical situations as in aquifers (Liu et al. 2017), in filters (Payri et al. 2011), near wellbores in hydrocarbon recovery or in column reactors (Enayati et al. 2018). A macroscopic empirical law classically employed to take into account inertial effects is the popular Darcy-Forchheimer momentum equation (Forchheimer 1901) given by

Didier Lasseux

didier.lasseux@u-bordeaux.fr

1 Arts et Métiers, CNRS, I2M, Esplanade des Arts et Métiers, 33405 Talence Cedex, France

2 CNRS, I2M, UMR 5295, Esplanade des Arts et Métiers, 33405 Talence Cedex, France 


$$
-\frac{\partial\left\langle p_{\beta}\right\rangle^{\beta}}{\partial x}=\alpha_{1}\left\langle v_{\beta}\right\rangle+\rho_{\beta} \alpha_{2}\left|\left\langle v_{\beta}\right\rangle\right|\left\langle v_{\beta}\right\rangle
$$

Note that in this expression, the pressure and velocity are understood in the sense of their intrinsic and superficial average, respectively, the definitions of which will be made clear in Sect. 2.1. Some fair agreement with the quadratic nature of the correction to the linear Darcy's law was found with numerical results obtained in 2D structures made of parallel and regularly arranged circular cylinders for flow orthogonal to the cylinders axes (Lee and Yang 1997). Reasonable agreement was also found in the turbulent regime for some special 2D and 3D geometries using a general RANS model (Soulaine and Quintard 2014). In addition, the form of Eq. (1) was assumed to be a valid macroscopic steady model which was used as the basis for a second upscaling in order to obtain a model operating in heterogeneous porous media (Auriault et al. 2007). However, validity of this steady model, in particular regarding the quadratic correction term, has been the object of an active research over the past 30 years (see for instance discussions in Lasseux et al. (2011), Lasseux and Valdés-Parada (2017) and references therein) yielding to the identification of several regimes. Nevertheless, the macroscale description of the physics which should formally result from an upscaling procedure keeps reminiscence of the microscale flow process through the inherent dependence of the macroscopic coefficients [ $\alpha_{1}$ and $\alpha_{2}$ in Eq. (1)] upon the microstructure of the porous material [see an example in Yazdchi et al. (2010)]. The analysis of the effect of the microstructure on the inertial correction, in particular its ordered character or not, was investigated in Lasseux et al. (2011) and the present work is a thorough continuation on the same lines. In addition, if a time-dependent flow is under concern, the question on the dependence of the transition to unsteadiness upon the microsctructure is even more acute.

Most works reported in the literature on this subject have adopted simple ordered or slightly disordered geometries, like, in 2D, a periodic pattern of parallel cylinders of circular or square cross section to determine the critical Reynolds number at which the flow becomes unsteady (Kawaguti 1955; Jackson 1987; Kumar and Mittal 2006; Lankadasu and Vengadesan 2008), to investigate the wake behavior (Gera et al. 2010; Kelkar and Patankar 1992), to study the interactions of wakes and evaluate the critical Reynolds number (Mizushima and Akinaga 2003 ) or to study the effects of cylinder spacing on flow (Kumar et al. 2008). There are also many studies using more complex pore-like geometries to investigate the flow properties. In the work by Amaral Souto and Moyne (1997), a regular square tessellation of the 2D unit cell used for the computational domain and a random distribution of the phase indicator (solid or fluid) on the resulting grid was performed to compute the inertial flow, yielding the confirmation of the cubic correction to Darcy's law in a weak inertia regime identified earlier (Barrère 1990; Mei and Auriault 1991). Similarly, a disordered structure was considered by Andrade et al. (1999) that was obtained by removing square obstacles from a $64 \times 64$ square lattice until a pore space with a prescribed porosity was generated. It was used to statistically characterize the transition from linear to nonlinear flow in terms of the distribution of kinetic energy. More recently, Lasseux et al. (2011) subdivided the periodic 2D domain under consideration into equal-sized unit cells with one solid inclusion of eventually random size placed randomly inside each unit cell. In this way, they studied the effects of locally disordered (LD) structures on the inertial correction to Darcy's law. Similar structures were adopted by Agnaou (2015), Agnaou et al. (2016, 2017) to study the first Hopf bifurcation for one-phase flow or to investigate the origin of inertial effects through a detailed investigation of the flow structure. 
Although many studies have been dedicated to the analysis of flow in different types of pore structures, a more systematic study on the effects of the microstructure on the onset and on the role of inertia, as well as on the occurrence of unsteadiness, is still lacking.

Based on the above considerations, the present study is a continuation of previous investigations (Abbasian Arani 2006; Lasseux et al. 2011; Agnaou 2015; Agnaou et al. 2017) taking into account six different types of 2D globally disordered (GD) structures with solid inclusions of circular or square cross sections. The focus is laid upon 2D configurations, keeping in mind that $3 \mathrm{D}$ cases are also of major interest, in particular with the objective of comparing the two situations and analyzing whether the fact that the inertial correction departs from the quadratic (Forchheimer) form can be reasonably attributed to 3D effects as suggested in Fourar et al. (2004). Compared to ordered and locally disordered (LD) structures studied in the above-cited references, the accent is laid upon much stronger disorder, considering random size distributions and orientations of the solid obstacles. Comparisons are made between GD structures and simple geometries mentioned above and a thorough analysis is carried out in order to investigate the effects of the microscopic pore structures on the flow regimes considering inertia.

The incipient departure from Darcy's law due to the contribution of inertia has been observed by many researchers (Edwards et al. 1990; Koch and Ladd 1997; Andrade et al. 1999; Lasseux et al. 2011; Agnaou et al. 2017) in the laminar flow regime prior to the appearance of turbulence. The flow becomes unsteady for a Reynolds number larger than a critical value in the laminar regime. However, in some works on inertial effects (Andrade et al. 1999; Chai et al. 2010; Lasseux et al. 2011), the flow was just assumed to be stationary in a limited Reynolds number range without a pre-determination of the critical Reynolds number. Therefore, in order to ensure a more rigorous and complete analysis in terms of the domain of validity of steady flow, the critical Reynolds number was first determined for the structures considered in this paper. The component of the average velocity orthogonal to the mean flow was monitored to capture flow unsteadiness. Then, steady numerical simulations were carried out to identify the different inertia regimes corresponding to weak, strong and beyond strong inertia (Agnaou et al. 2017) as well as to further assess the effects of pore structure disorder on the inertial contributions to the deviations from Darcy's law.

The paper is organized as follows. Section 2 is dedicated to a presentation of the microand macroscopic models for incompressible flow and the different pore structures used to carry out the simulations together with the numerical procedure are detailed. Results are reported in Sect. 3 with, successively, the critical Reynolds number (Sect. 3.1), the influence of the microstructure on the permeability (Sect. 3.2.1) and on the flow regimes and inertia (Sect. 3.2.2). Conclusions are drawn in Sect. 4.

\section{Physical Models: Methodology and Tools}

In this section, the governing equations for stationary isothermal flow of an incompressible Newtonian fluid ( $\beta$-phase) in a porous medium at the microscale and at the macroscale are first recalled, together with the closure problem relating the two, and the inertial correction vector on which discussions will be based is introduced. The six types of pore structures used to analyze the flow are also listed, with a specific nomenclature in order to clearly distinguish them from those considered in previous studies. In addition, numerical algorithms adopted for the two softwares employed in this work are briefly introduced. 


\subsection{Governing Equations: Microscopic and Macroscopic Models}

Model porous media under consideration are periodic 2D structures so that the analysis can be restricted to a periodic unit cell, $\mathscr{V}$, representative of the process. For convenient comparison with existing reported results, the governing mass (continuity) and momentum (Navier-Stokes) conservation equations, together with the periodic boundary conditions, are written in a dimensionless form, yielding the following initial boundary value problem in this periodic domain (Lasseux et al. 2011; Agnaou et al. 2016)

$$
\begin{aligned}
& R e^{*}\left(\frac{\partial \mathbf{v}_{\beta}^{*}}{\partial t^{*}}+\mathbf{v}_{\beta}^{*} \cdot \nabla \mathbf{v}_{\beta}^{*}\right)=-\nabla \tilde{p}_{\beta}^{*}+\nabla^{2} \mathbf{v}_{\beta}^{*}-\nabla\left\langle p_{\beta}^{*}\right\rangle^{\beta} \text { in } \mathscr{V}_{\beta} \\
& \nabla \cdot \mathbf{v}_{\beta}^{*}=0 \text { in } \mathscr{V}_{\beta} \\
& \mathbf{v}_{\beta}^{*}=\mathbf{0} \text { at } \Lambda_{\beta \sigma} \\
& \mathbf{v}_{\beta}^{*}=0 \text { at } t^{*}=0 \text { in } \mathscr{V}_{\beta} \\
& \left\langle\tilde{p}_{\beta}^{*}\right\rangle^{\beta}=0 t^{*}>0 \text { in } \mathscr{V}_{\beta} \\
& \mathbf{v}_{\beta}^{*}\left(\mathbf{r}^{*}+\mathbf{l}_{i}^{*}\right)=\mathbf{v}_{\beta}^{*}\left(\mathbf{r}^{*}\right) \quad \tilde{p}_{\beta}^{*}\left(\mathbf{r}^{*}+\mathbf{l}_{i}^{*}\right)=\tilde{p}_{\beta}^{*}\left(\mathbf{r}^{*}\right) \quad i=1,2,3
\end{aligned}
$$

where, for the sake of simplicity and consistent comparison with existing results, no body force was taken into account. Here, $\mathscr{V}_{\beta}$ and $\Lambda_{\beta \sigma}$ represent the volume of the $\beta$-phase contained in $\mathscr{V}$ and the fluid-solid $(\beta-\sigma)$ interface, respectively; $\mathbf{v}_{\beta}^{*}, p_{\beta}^{*}$ are the dimensionless $\beta$-phase velocity and pressure. Time and position variables are denoted $t^{*}$ and $\mathbf{r}^{*}$ and $\mathbf{l}_{i}^{*}(i=1,2,3)$ represents the dimensionless periodic lattice vectors of the unit cell. The pressure $p_{\beta}^{*}$ in Eq. (2a) has been replaced by its expression given by Gray's decomposition (Gray 1975) in terms of the intrinsic average pressure, $\left\langle p_{\beta}^{*}\right\rangle^{\beta}$, and its deviation, $\tilde{p}_{\beta}^{*}$, namely

$$
p_{\beta}^{*}=\left\langle p_{\beta}^{*}\right\rangle^{\beta}+\tilde{p}_{\beta}^{*}
$$

where the intrinsic average of any quantity $\psi$ in $\mathscr{V}_{\beta}$ is defined as

$$
\langle\psi\rangle^{\beta}=\frac{1}{V_{\beta}} \int_{\mathscr{V}_{\beta}} \psi \mathrm{d} V=\epsilon^{-1}\langle\psi\rangle
$$

$\epsilon$ denoting the porosity of the structure and $\langle\psi\rangle$ the superficial average of $\psi$. The decomposition in Eq. (3) allows to impose periodicity for the pressure deviation, while considering $\nabla\left\langle p_{\beta}^{*}\right\rangle^{\beta}$ as a driving force. Dimensionless pressure and velocity are defined from their dimensional counterparts using the reference values $v_{\text {ref }}=\frac{l^{2}}{\mu_{\beta}}\left|\nabla\left\langle p_{\beta}\right\rangle^{\beta}\right|$ and $p_{\text {ref }}=l\left|\nabla\left\langle p_{\beta}\right\rangle^{\beta}\right|$, yielding $\left|\nabla\left\langle p_{\beta}^{*}\right\rangle^{\beta}\right|=1$ so that any flow can be obtained from an appropriate independent choice of the Reynolds number and orientation of $\nabla\left\langle p_{\beta}^{*}\right\rangle^{\beta}$ (Lasseux et al. 2011). The Reynolds number $R e^{*}$ in Eq. (2a) is defined by:

$$
R e^{*}=\frac{\rho_{\beta} v_{\text {ref }} l}{\mu_{\beta}}=\frac{\rho_{\beta} l^{3}}{\mu_{\beta}^{2}}\left|\nabla\left\langle p_{\beta}\right\rangle^{\beta}\right|
$$

In the above equations, $l$ represents the size of the periodic geometrical unit cell in the case of ordered structures (see Fig. 1a and b) or, in the case of locally and globally disordered structures, the size of the periodic unit cell divided by the square root of $n, n$ being the number of obstacles in this unit cell (see Figs. $1 \mathrm{c}$ and d, 2). In addition, $\rho_{\beta}$ and $\mu_{\beta}$ are the density and dynamic viscosity of the $\beta$-phase. 
Under the assumptions of quasisteady flow, a scale hierarchy $(l \ll L$ or $\sqrt{n} l \ll L, L$ being the size of the macroscopic medium considered as a periodic structure in its homogeneous part) and $\frac{\rho_{\beta}\left|\left\langle\mathbf{v}_{\beta}\right\rangle^{\beta}\right| d_{p}}{\mu_{\beta}} \ll L / d_{p}$ where $d_{p}$ is the characteristic pore size (Whitaker 1969, 1996; Lasseux et al. 2011), the pore-scale boundary value problem can be upscaled to yield the macroscopic model using the volume averaging method (Whitaker 1996, 1999). In the dimensionless form, it is given by (Lasseux et al. 2011)

$$
\begin{aligned}
& \nabla \cdot\left\langle\mathbf{v}_{\beta}^{*}\right\rangle=0 \text { in } \mathscr{V} \\
& \left\langle\mathbf{v}_{\beta}^{*}\right\rangle=-\mathbf{K}^{*} \cdot \nabla\left\langle p_{\beta}^{*}\right\rangle^{\beta}-\mathbf{F} \cdot\left\langle\mathbf{v}_{\beta}^{*}\right\rangle \text { in } \mathscr{V}
\end{aligned}
$$

valid far enough from the macroscopic boundaries nearby which the scale hierarchy fails. The dimensionless intrinsic permeability, $\mathbf{K}^{*}=\mathbf{K} / l^{2}$, and the Darcy's law correction tensor, $\mathbf{F}$, can be determined by solving the associated closure problem for $R e^{*}=0$ and for any prescribed value of $R e^{*}$ respectively. In its dimensionless form, this closure problem is given by

$$
\begin{aligned}
& R e^{*} \mathbf{v}_{\beta}^{*} \cdot \nabla \mathbf{M}^{*}=-\nabla \mathbf{m}^{*}+\nabla^{2} \mathbf{M}^{*}+\mathbf{I} \text { in } \mathscr{V}_{\beta} \\
& \nabla \cdot \mathbf{M}^{*}=\mathbf{0} \text { in } \mathscr{V}_{\beta} \\
& \mathbf{M}^{*}=\mathbf{0} \text { at } \Lambda_{\beta \sigma} \\
& \left\langle\mathbf{m}^{*}\right\rangle^{\beta}=\mathbf{0} \\
& \mathbf{m}^{*}\left(\mathbf{r}^{*}+\mathbf{l}_{i}^{*}\right)=\mathbf{m}^{*}\left(\mathbf{r}^{*}\right) \quad \mathbf{M}^{*}\left(\mathbf{r}^{*}+\mathbf{l}_{i}^{*}\right)=\mathbf{M}^{*}\left(\mathbf{r}^{*}\right) \quad i=1,2,3
\end{aligned}
$$

where $\mathbf{m}^{*}$ and $\mathbf{M}^{*}$ are closure variables that map $\left\langle\mathbf{v}_{\beta}^{*}\right\rangle$ onto $\tilde{p}^{*}$ and $\tilde{\mathbf{v}}^{*}$ respectively (Whitaker 1996). It must be noticed that an alternative form of the inertial term in Eq. (7a) can be used with the advantage of being fully closed in contrast with the expression $\operatorname{Re}^{*} \mathbf{v}_{\beta}^{*} \cdot \nabla \mathbf{M}^{*}$ which seems to indicate that $\mathbf{v}_{\beta}^{*}$ is required to solve this closure problem. In fact, as shown in ValdésParada et al. (2016), the left-hand side of Eq. (7a) can be written as $R e^{*} \nabla\left\langle p_{\beta}^{\star}\right\rangle^{\beta} \cdot \mathbf{M}^{\star T} \cdot \nabla \mathbf{M}^{*}$, with the drawback, however, of introducing a strong nonlinearity which makes the closure problem more complex to solve. From this closure, it follows that

$$
\left\langle\mathbf{M}^{*}\right\rangle=\mathbf{H}^{*}
$$

with $\left\langle\mathbf{M}^{*}\right\rangle=\mathbf{K}^{*}$ when $R e^{*}=0$, and

$$
\mathbf{F}=\mathbf{K}^{*} \cdot \mathbf{H}^{*-1}-\mathbf{I}
$$

\subsection{Determination of the Inertial Correction}

The inertial correction to Darcy's law is given by $-\mathbf{F} \cdot\left\langle\mathbf{v}_{\beta}^{*}\right\rangle$ as can be seen in Eq. (6b). For convenience, a renormalization is applied leading to express the correction as a dimensionless vector, $\mathbf{f}_{c}$, as [see Lasseux et al. (2011) for the details and the physical interpretation]

$$
\mathbf{f}_{c}=\frac{-\mathbf{F} \cdot\left\langle\mathbf{v}_{\beta}^{*}\right\rangle}{\left|\left\langle\mathbf{v}_{\beta}^{*}\right\rangle\right|}
$$

An alternative expression can be employed, avoiding the computation of the tensor $\mathbf{F}$, and this is obtained by making use of Eq. (6b), which yields

$$
\mathbf{f}_{c}=\frac{\left\langle\mathbf{v}_{\beta}^{*}\right\rangle+\mathbf{K}^{*} \cdot \nabla\left\langle p_{\beta}^{*}\right\rangle^{\beta}}{\left|\left\langle\mathbf{v}_{\beta}^{*}\right\rangle\right|}
$$




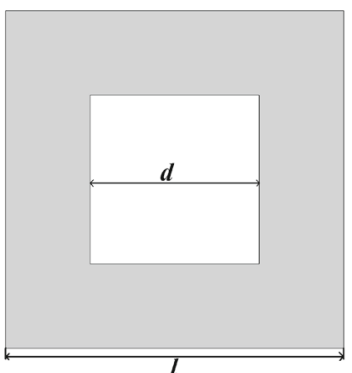

(a) $\mathrm{OS}$

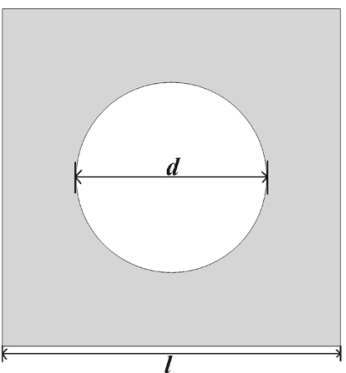

(b) $\mathrm{OC}$

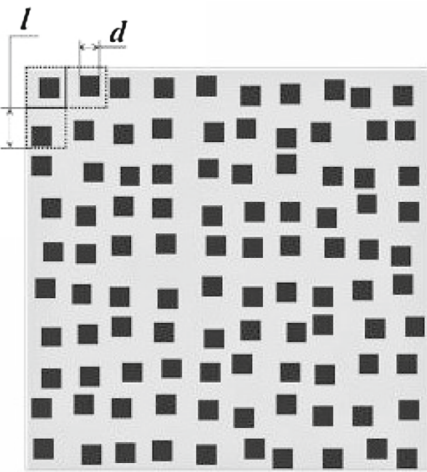

(c) LWD

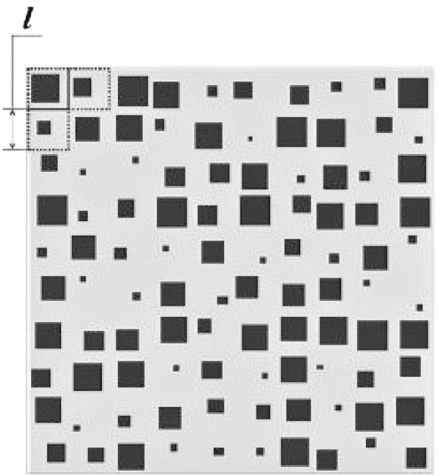

(d) LSD

Fig. 1 Ordered (a, b) and locally disordered (c, d) pore structures (Lasseux et al. 2011; Agnaou et al. 2016). OS, OC, LWD and LSD, respectively, refer to ordered squares, ordered circles, locally weakly disordered and locally strongly disordered structures.

Under this form, the determination of $\mathbf{f}_{\mathbf{c}}$ only requires the solution of the flow within the unit cell resulting from $\nabla\left\langle p_{\beta}^{*}\right\rangle^{\beta}$ and a given Reynolds number, together with the permeability tensor. Rather than $R e^{*}$, a Reynolds number, depending only on macroscopic quantities is used in the present work that is given by:

$$
R e_{k}=\frac{\rho_{\beta}\left|\left\langle\mathbf{v}_{\beta}\right\rangle\right| \sqrt{k}}{\mu_{\beta}}=\left|\left\langle\mathbf{v}_{\beta}^{*}\right\rangle\right| \sqrt{k^{*}} R e^{*}
$$

where $k^{*}=k / l^{2}$ is the dimensionless intrinsic permeability considering an isotropic structure. It should be noted that a Reynolds number based on the grain size of the porous medium and the average velocity is also widely employed in the literature (see a discussion on the different definitions of the Reynolds number in Lasseux et al. (2011), section II.B), namely

$$
\operatorname{Re}_{d}=\frac{\rho_{\beta}\left|\left\langle\mathbf{v}_{\beta}\right\rangle\right| d}{\mu_{\beta}}=\left|\left\langle\mathbf{v}_{\beta}^{*}\right\rangle\right| d^{*} R e^{*}
$$

where $d^{*}=d / l$ is the dimensionless grain size of the porous structure (Fig. 1). For the random structures used in this work, $d$ was estimated as the quadratic mean of the obstacle sizes, i.e., $d=\sqrt{\sum_{i=1}^{i=n} d_{i}^{2} / n}$.

From the above expression of $\mathbf{f}_{\mathbf{c}}$, it appears that when the correction to Darcy's law, $-\mathbf{F} \cdot\left\langle\mathbf{v}_{\beta}^{*}\right\rangle$, scales as $\left|\left\langle\mathbf{v}_{\beta}^{*}\right\rangle\right|^{3}$ (respectively $\left|\left\langle\mathbf{v}_{\beta}^{*}\right\rangle\right|^{2}$ ) as expected in the weak (respectively 


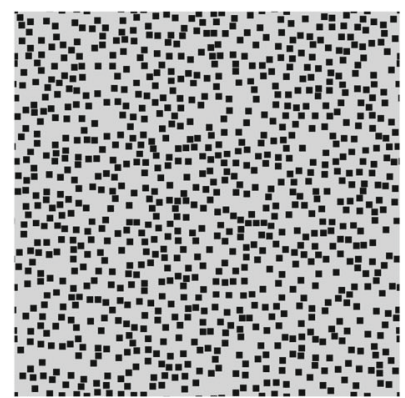

(a) GWD

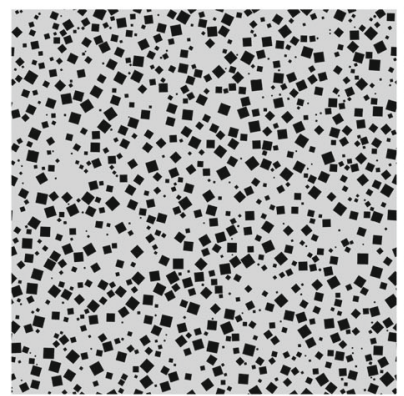

(d) GSDf

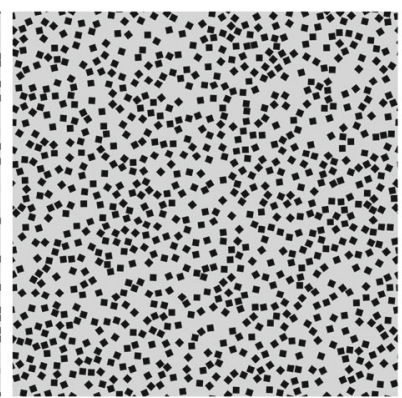

(b) GWDf

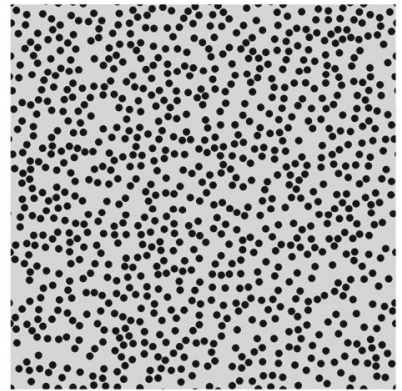

(e) GWDC

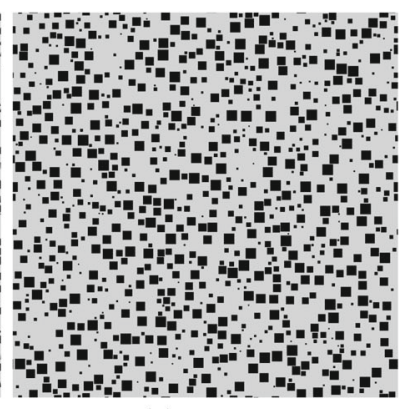

(c) GSD

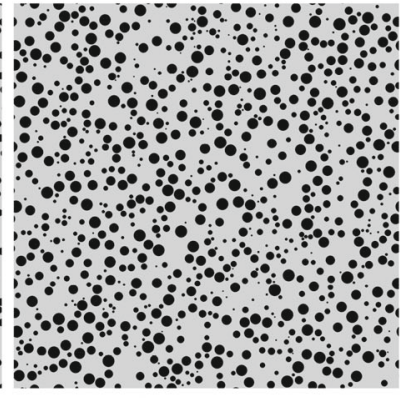

(f) GSDC

Fig. 2 Examples of generated periodic unit cells of globally disordered pore structures including 900 solid obstacles

strong) inertia regime (Lasseux et al. 2011), then $\mathbf{f}_{\mathbf{c}}$ depends linearly on $R e_{k}^{2}$ (respectively $R e_{k}$ ). Therefore, in this paper, the interval of validity of each regime will be identified from the relationship between $\mathbf{f}_{c}$ and $R e_{k}$.

\subsection{Pore Structures}

The nomenclature of the disordered structures considered in this work shall be first briefly introduced in order to clearly distinguish them. The random porous media studied in previous works (Lasseux et al. 2011; Agnaou et al. 2016, 2017) are referred here to as locally weakly disordered (LWD, Fig. 1c) and locally strongly disordered (LSD, Fig. 1d) porous media.

Four other globally disordered (GD) 2D porous media, generated with square solid inclusions, are considered in this study, namely globally weakly disordered structures (GWD, Fig. 2a), globally weakly disordered structures with flipped squares (GWDf, Fig. 2b), globally strongly disordered structures (GSD, Fig. 2c), globally strongly disordered structures with flipped squares (GSDf, Fig. 2d). Two additional ones with circular cylinders are also considered, namely globally weakly and strongly disordered structures with circular cylinders (respectively, GWDC, Fig. 2e and GSDC, Fig. 2f). Weakly disordered structures refer to cases for which inclusions are of uniform size but are randomly placed within the unit cell while, for strong disorder, inclusion size is also randomly chosen. In contrast with the local disorder envisaged so far for which random placement was spatially confined within a region of size $l$, global disorder allows a random distribution of the inclusions within the unit cell of size $\sqrt{n} l$. All the GD structures have a dimensionless size of $30 \times 30$, which means $n=900$. 
Indeed, in accordance with a previous study (Agnaou et al. 2017), this size (and number of inclusions) is sufficiently large to reduce artifacts related to periodic boundary conditions (Hill and Koch 2002). For comparison purposes, the value of the porosity $(\epsilon=0.75)$ is kept the same as in previous works (Lasseux et al. 2011; Agnaou et al. 2017). In order to avoid overlapping of the cylinders and extremely narrow channels that would locally require an exceedingly large number of grid blocks, the following constraint was imposed during generation: inclusions were randomly distributed imposing a minimum pore throat size of 0.1 times the average edge size (for square inclusions) or diameter (for circular inclusions).

\subsection{Numerical Tools and Procedure}

In order to identify the critical Reynolds number corresponding to the first Hopf bifurcation, transient flow was first simulated in all the geometries generated in this work. This was performed by solving Eq. (2) using the transient solver icoFoam, which is based on the Pressure Implicit Split Operator (PISO) algorithm in the OpenFOAM package (Weller et al. 1998). The linear system solver for the velocity is based on the Preconditioned Bi-Conjugate Gradient (PBiCG) with a tolerance of $10^{-10}$, while, for the pressure, the Preconditioned Conjugate Gradient (PCG) solver was chosen with a tolerance of $10^{-7}$. These tolerances were selected to accurately determine the critical Reynolds number intervals and are in agreement with those employed in a previous work (Agnaou et al. 2016). The time step was calculated according to the mesh size and the velocity magnitude in order to satisfy the Courant-Friedrichs-Lewy (CFL) stability condition. The Courant number was always smaller than 0.5 in this work.

Once the critical Reynolds number delimiting the steady laminar flow regime was identified, numerical simulations of the steady incompressible flow were carried out in all the generated pore geometries, allowing a detailed analysis of the inertial correction to Darcy's law. To do so, steady-state computations were performed within the Reynolds number range that ensures steady laminar flow for which the average model in Eq. (6) is applicable. This was achieved by making use of the partial differential equation (PDE) module in the COMSOL multiphysics commercial software package (COMSOL Multiphysics 2012). The system of nonlinear equations was discretized and linearized around an initial estimate using the COMSOL stationary solver. The system of linearized equations was solved with the direct solver MUMPS (Multifrontal Massively Parallel Direct Sparse Solver) (Amestoy et al. 2001), which makes use of a LU factorization. The computational domain was discretized employing unstructured triangular elements with local mesh refinement in constricted zones as illustrated in Fig. 3. The average pressure gradient was such that $\nabla\left\langle p_{\beta}^{*}\right\rangle^{\beta}=\mathbf{e}_{x}$ and the mean flow is expected to be aligned with the driving force due to the isotropic character of the random structures.

\section{Results and Analysis}

The hydrodynamics within the spatially periodic porous media under consideration is analyzed in order to investigate the effect of the microscopic structure on the inertial contribution to the deviation from Darcy's law. In order to ensure that results are representative of the physics at play in the corresponding structure of infinite extent and circumvent the fact that the size of the random unit cell may not be a representative domain of the medium, an ensemble average of results obtained for ten realizations of each structure was considered, except for 


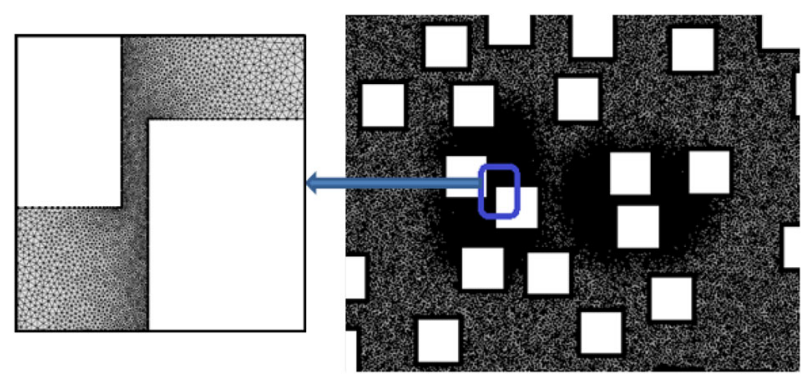

Fig. 3 Example of locally refined mesh

the determination of the critical Reynolds number (see details below). According to previous studies (Abbasian Arani 2006; Lasseux et al. 2011; Agnaou 2015), this choice represents a good compromise between computational tractability and accuracy requirements.

Before proceeding any further, it is of importance to study mesh convergence for the numerical solutions (Chai et al. 2010). This analysis is reported in the "Appendix."

\subsection{Determination of the Critical Reynolds Number}

Transition from steady to time-periodic flow within the laminar regime is marked by a Hopf bifurcation (Marsden and McCracken 1976) characterized by a critical value, $R e_{\mathrm{c}}^{*}$ (or $R e_{d c}$ or $R e_{k c}$ ), of the Reynolds number. For flow past an isolated circular cylinder, the wake behind the cylinder becomes periodically oscillating at the bifurcation which occurs at $R e_{d c} \approx 50$, leading to the formation of a Karman vortex street (Jackson 1987). In many engineering fields, the occurrence of the periodic vortex shedding is believed to result in the failure of structures exposed to this type of flow (Franke et al. 1990). Here, the determination of this critical value is aimed at restricting our investigations of inertial effects to the steady regime.

Capturing the occurrence of unsteadiness in the random structures under consideration is not an easy task a priori. The best indicator was found to be the time evolution of the $y$-component of the average dimensionless velocity, $\left\langle v_{\beta y}^{*}\right\rangle$, and this was further checked to be in agreement with the occurrence of unsteadiness of the pointwise $y$-velocity component at particular locations in the pore space. Simulations of unsteady flow were carried out over a sufficiently long time for successive increasing Reynolds number values until oscillatory flow was observed. It should be emphasized that, unlike other works in the literature where a disturbance was introduced into the simulations (Kelkar and Patankar 1992; Chen et al. 1995), no perturbation was added in this study and the periodic oscillation is purely due to the physical instability of the problem, depending on the pore geometries and Reynolds number. To keep the simulation time reasonable, the critical Reynolds number values were estimated on only two distinct realizations of each structure.

As an illustration, evolutions of $\left\langle v_{\beta y}^{*}\right\rangle$ obtained on a GWDf realization for $R e^{*}=$ $12,000\left(R e_{k}=5.48, R e_{d}=26.74\right)$ and $R e^{*}=14,000\left(R e_{k}=6, R e_{d}=29.31\right)$ are reported in Fig. 4a and c respectively. For $R e^{*}=12,000,\left\langle v_{\beta y}^{*}\right\rangle$ remains constant over time after an initial transient period, showing that the flow stabilizes in a steady regime. Conversely, for $R e^{*}=14,000,\left\langle v_{\beta y}^{*}\right\rangle$ exhibits a permanent oscillatory behavior, typical of a post-bifurcation regime. This is further confirmed by the Fourier spectra represented versus the Strouhal number, $S t$, defined as $S t=\frac{f l}{v_{\text {ref }}}=\frac{\mu_{\beta} f}{l\left|\nabla\left\langle p_{\beta}\right\rangle^{\beta}\right|}, f$ being the frequency of the oscillation, in Fig. $4 \mathrm{~b}$ and d, respectively. These two figures show that $\left\langle v_{\beta y}^{*}\right\rangle(t)$ has a zero frequency 


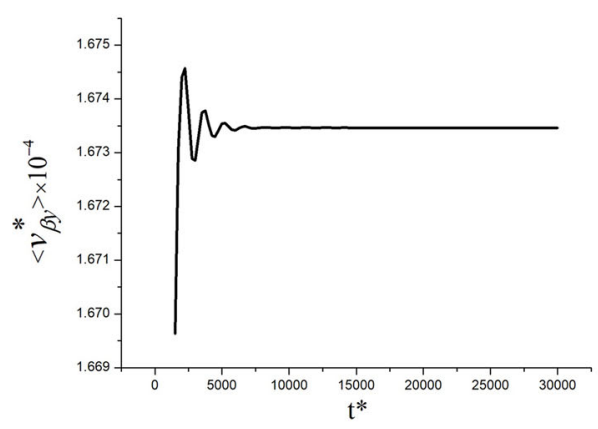

(a)

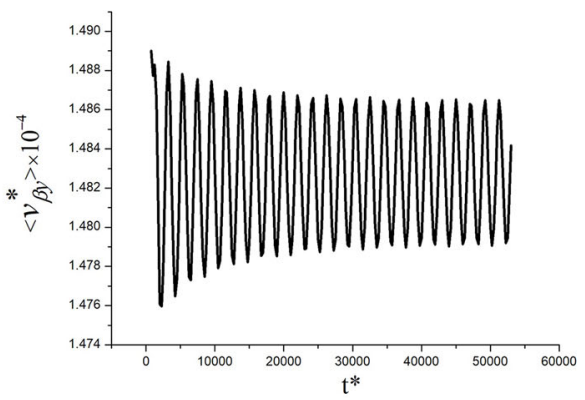

(c)

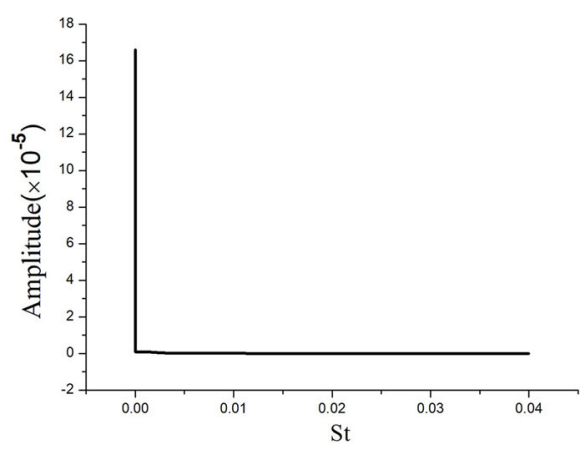

(b)

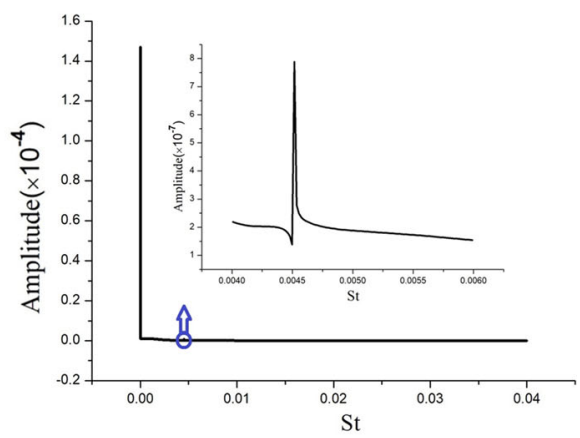

(d)

Fig. 4 Time evolution of the $y$-component of the average velocity $(\mathbf{a}, \mathbf{c})$ and corresponding Fourier spectra $(\mathbf{b}, \mathbf{d})$ for a GWDf realization. $R e^{*}=12,000\left(R e_{k}=5.48, R e_{d}=26.74\right)(\mathbf{a}, \mathbf{b})$ and $R e^{*}=14,000\left(R e_{k}=\right.$ $\left.6, R e_{d}=29.31\right)(\mathbf{c}, \mathbf{d})$

at $R e^{*}=12,000$ and is characterized by a unique frequency for $R e^{*}=14,000$. As $R e^{*}$ increases, additional nonzero frequencies are observed in the spectra and the amplitudes of the corresponding signals also increase in accordance with previously reported results (Agnaou et al. 2016). As a consequence, the critical Reynolds number for the particular case of Fig. 4 can be identified to be $R e_{c}^{*}=13,000 \pm 1000\left(R e_{k c}=5.75 \pm 0.26, R e_{d c}=28.05 \pm 1.28\right)$.

Critical Reynolds numbers for the different structures under consideration in this work are listed in Table 1. Values of $R e_{d c}$ and $R e_{k c}$ are computed from the values of $R e_{\mathrm{c}}^{*}$ after the simulation is completed using Eqs. (12) and (13). Several conclusions on the effect of the geometry on $R e_{d c}$ can be drawn. Firstly, as has been pointed out by Agnaou et al. (2016), the critical Reynolds number, $R e_{d c}$, decreases drastically as more disorder is introduced into the pore structure. This can be observed from the values of $R e_{d c}$ obtained for all globally disordered structures (GWD, GWDf, GSD and GWDC) compared to those reported for locally disordered (LWD an LWDC) and ordered (OS and OC) media. Secondly, the effect of the size distribution of solid squares or circular obstacles is quite limited, as can be seen from the values of $R e_{d c}$ obtained for GWD and GSD structures. For this reason, critical values of the Reynolds number are not listed for GSDf and GSDC structures as they are expected to be similar to those obtained for GWDf and GWDC structures. Thirdly, unlike the study of Sohankar et al. (1998), in which the angle of incidence of the flow with respect to the square obstacle has a great influence on the critical Reynolds number, there is little effect of the square inclinations as can be seen from $R e_{d c}$ values for GWD and GWDf structures. 
Table 1 Critical Reynolds numbers $\left(R e_{d c}, R e_{k c}, R e_{c}^{*}\right)$ for GD structures and comparisons with results reported in previous works for ordered and LWD structures (Agnaou et al. 2016, 2017)

\begin{tabular}{llll}
\hline Structure & $R e_{d c}$ & $R e_{k c}$ & $R e_{c}^{*}$ \\
\hline GWD(1) & $36.12 \pm 1.26$ & $7.67 \pm 0.27$ & $17,000 \pm 1000$ \\
GWD(2) & $31.96 \pm 1.37$ & $6.79 \pm 0.29$ & $14,000 \pm 1000$ \\
GWDf(1) & $35.7 \pm 1.08$ & $7.39 \pm 0.22$ & $19,000 \pm 1000$ \\
GWDf(2) & $28.05 \pm 1.28$ & $5.75 \pm 0.26$ & $13,000 \pm 1000$ \\
GSD(1) & $27.31 \pm 1.95$ & $6.28 \pm 0.45$ & $9000 \pm 1000$ \\
GSD(2) & $37.11 \pm 1.18$ & $8.24 \pm 0.26$ & $18,000 \pm 1000$ \\
GWDC(1) & $41.16 \pm 2.28$ & $8.57 \pm 0.47$ & $11,000 \pm 1000$ \\
GWDC(2) & $38.21 \pm 2.34$ & $7.73 \pm 0.47$ & $11,000 \pm 1000$ \\
LWD (Agnaou et al. 2016) & $83.51 \pm 2.45$ & $19.15 \pm 0.56$ & 30,000 \\
LWDC (Agnaou et al. 2017) & 93.46 & 19.87 & 30,000 \\
OS (Agnaou et al. 2016) & $100.82 \pm 2.5$ & $23.02 \pm 0.57$ & $17,250 \pm 500$ \\
OC (Agnaou et al. 2017) & 101.39 & 21.09 & 17500 \\
\hline
\end{tabular}

This contrast may be attributed to the fact that, in the present study, randomness of the angle of incidence from $0^{\circ}$ to $45^{\circ}$ applied on the squares may lead to strong interactions of the wakes behind neighboring obstacles which may weaken the effects of the local flow incidence. Fourthly, $R e_{d c}$ values for structures composed of circular obstacles are slightly larger than those for structures with solid squares, as can be observed from a comparison of $R e_{d c}$ results obtained for GWD, LWD and OS structures on the one hand and for GWDC, LWDC and OC structures on the other hand. Finally, although the observation is restricted to only two realizations, a significant dispersion of the critical Reynolds number over the different realizations of the same structure seems to occur. A more detailed analysis, however beyond the scope of this work, would be necessary to quantify this effect. It should be noted that the above conclusions drawn for $R e_{d c}$ cannot be straightforwardly extended to $R e_{k c}$ or $R e_{c}^{*}$, since for instance, $R e_{d}$ and $R e_{k}$ may have opposite variations, as can be seen from the results between GSD(2) and GWDC(2) or between OS and OC. Indeed, different definitions of the Reynolds number may lead to opposite conclusions as pointed out by Lasseux et al. (2011). In the following sections, analysis of the inertial correction is restricted to $R e_{k}$ up to the lower bound value obtained for each structure for which steady flow is ensured.

\subsection{Influence of the Microstructure}

The microstructure has an impact not only on the permeability of the medium but also on the inertial regimes and the ranges of $R e_{k}$ on which they can be identified.

\subsubsection{On the Permeability}

The permeability tensor, which represents an important physical parameter for the process under study as it is further used to compute $\mathbf{f}_{c}$, is analyzed in this section. The attention is focussed on the mean diagonal term $k^{*}=\frac{\overline{k_{x x}^{*}}+\overline{k_{y y}^{*}}}{2}$ and on the extra diagonal term $\overline{k_{x y}^{*}}=\overline{k_{y x}^{*}}$, the overbar indicating an average over the ten realizations. Values of these two quantities are reported in Table 2 together with some previously reported results obtained on ordered and 
Table 2 Average values of the components of $\mathbf{K}^{*}$ obtained over ten realizations of GD structures and comparisons with previous works (Lasseux et al. 2011; Agnaou et al. 2017)

\begin{tabular}{lll}
\hline Structure & $k^{*}(\sigma)$ & $\overline{k_{x y}^{*}}(\sigma)$ \\
\hline GWD & $0.01123(3.7380 \mathrm{e}-4)$ & $-1.50624 \mathrm{e}-5(1.82847 \mathrm{e}-4)$ \\
GWDf & $0.01039(2.5727 \mathrm{e}-4)$ & $2.90673 \mathrm{e}-5(2.05526 \mathrm{e}-4)$ \\
GSD & $0.01285(4.71718 \mathrm{e}-4)$ & $3.08100 \mathrm{e}-4(2.30627 \mathrm{e}-4)$ \\
GSDf & $0.01231(3.47639 \mathrm{e}-4)$ & $8.133 \mathrm{e}-5(2.28609 \mathrm{e}-4)$ \\
GWDC & $0.01367(5.20535 \mathrm{e}-4)$ & $3.85605 \mathrm{e}-4(2.33563 \mathrm{e}-4)$ \\
GSDC & $0.01547(4.4480 \mathrm{e}-4)$ & $3.47906 \mathrm{e}-4(2.334833 \mathrm{e}-4)$ \\
LWD (Lasseux et al. 2011) & $-1.01 \mathrm{e}-4(4.75 \mathrm{e}-4)$ \\
LWD (Agnaou et al. 2017) & $0.01308(3.82 \mathrm{e}-4)$ & $-3.43 \mathrm{e}-4(6.69 \mathrm{e}-4)$ \\
LSD (Lasseux et al. 2011) & 0.01321 & \\
LSD (Agnaou et al. 2017) & $0.01350(7.72 \mathrm{e}-4)$ & \\
OS (Lasseux et al. 2011) & 0.01440 & \\
OS (Agnaou et al. 2017) & 0.01304 & \\
OC (Agnaou et al. 2017) & 0.01303 & \\
\hline
\end{tabular}

${ }^{a}$ Results were obtained with $n=100$ and 10 realizations

${ }^{\mathrm{b}}$ Results were obtained with $n=900$ and one realization

locally disordered structures (Lasseux et al. 2011; Agnaou et al. 2017). Two major remarks must be made on these results. First, the extra diagonal term remains approximately 2 to 3 orders of magnitude smaller than the diagonal term, confirming that the structures are isotropic and justifying the use of $k^{*}$ to characterize their permeability. Secondly, as mentioned in the appendix, the standard deviation, $\sigma$, on the diagonal term is roughly 100 times smaller than $k^{*}$. This confirms that the ensemble average provides an accurate representation of the infinite medium for each structure. In addition, results in Table 2 also show that the two GD structures containing circular cylinders (GWDC and GSDC) are more permeable than those containing square cylinders, confirming the same tendency observed on ordered structures (Agnaou et al. 2016). This result can be explained by the fact that local variations in the direction of flow streamlines are more abrupt in the presence of square obstacles than for circular inclusions, yielding a stronger resistance to flow. Moreover, $k^{*}$ for GD structures made up of square cylinders is slightly smaller than that obtained on corresponding less disordered geometries, namely OS, LWD and LSD. This results from the fact that the randomly distributed obstructions reduce the possibility of the formation of 'preferential channels,' confirming an observation reported elsewhere (Clavier 2015). As a final remark, one may observe that structures made up of square obstacles have a permeability which, in the increasing order, can be classified as follows: GWDf, GWD, GSDf, GSD, OS, LWD and LSD.

\subsubsection{On the Flow Regimes and Inertia}

In Fig. 5, the two components, $f_{c x}$ and $f_{c y}$, of the correction vector to Darcy's law (see Eq. 11) obtained with $\nabla\left\langle p_{\beta}^{*}\right\rangle^{\beta}=\mathbf{e}_{x}$ are plotted as functions of $R e_{k}$ for the GWD and GWDf structures. A similar behavior was obtained for the other structures and is not represented here for the sake of brevity. It must be noted that $f_{c y} \approx 0$ and is hence completely negligible compared to $f_{c x}$, confirming that the structures remain isotropic in the inertial regime. As 


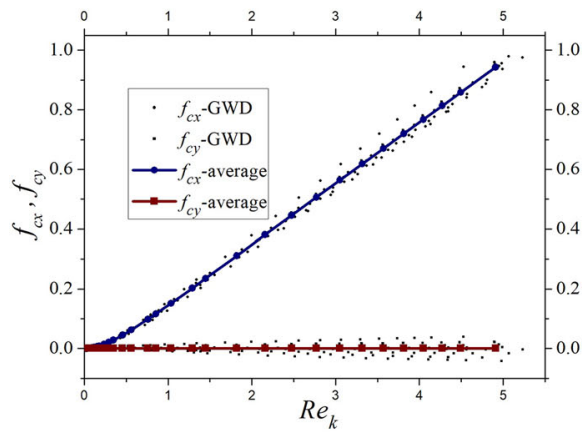

(a) GWD

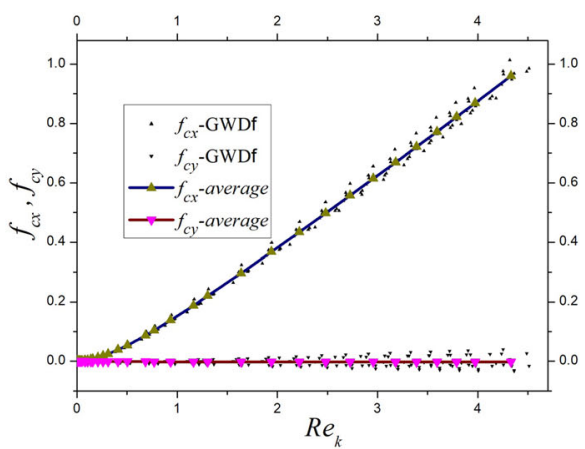

(b) GWDf

Fig. 5 Variations of the $x$ - and $y$-components of $\mathbf{f}_{c}$ with $R e_{k}$ for the ten realizations and their averages for the GWD and GWDf structures

Fig. 6 Variation of $f_{c x}$ versus $R e_{k}$, for GD structures and comparison with OS, OC, LWD and LWDC results from the literature (Lasseux et al. 2011; Agnaou et al. 2017)

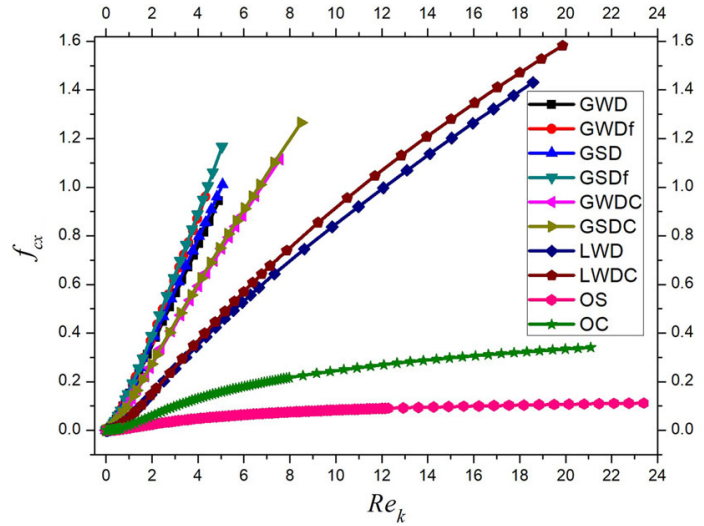

a consequence the analysis of the different flow regimes can be carried out on $f_{c x}$ only, in agreement with results for simpler geometries reported in the work of Lasseux et al. (2011). The variations of $f_{c x}$ versus $R e_{k}$ are represented in Fig. 6 for the different GD structures under consideration along with results on ordered and LD structures reported earlier (Lasseux et al. 2011; Agnaou et al. 2017). Data points are average values over all the realizations for each structure.

The dependence of $f_{c x}$ on $R e_{k}$ allows to distinguish the so-called weak inertia and strong inertia regimes (Skjetne and Auriault 1999; Lasseux et al. 2011) for which linear correlations versus $R e_{k}^{2}$ and $R e_{k}$ are applicable, respectively, corresponding to a cubic and quadratic dependence of the correction to the Darcy velocity in a dimensional form. This is highlighted in Fig. 7 representing these correlations on Reynolds number intervals where the Pearson coefficient, $R^{2}$, remains $\geq 0.9999$. Data points used for the fits and represented in this figure were obtained from an average of all the numerical results obtained on the ten realizations for each structure. Reynolds values intervals corresponding to the two regimes are indicated in Table 3 together with those obtained for LWD and ordered structures reported in Agnaou (2015). Obviously, the interval of the weak inertia regime shrinks as more disorder is introduced, confirming an observation already made (Lasseux et al. 2011). This may consistently explain why this regime is not easily identified during experimental observations on natural 


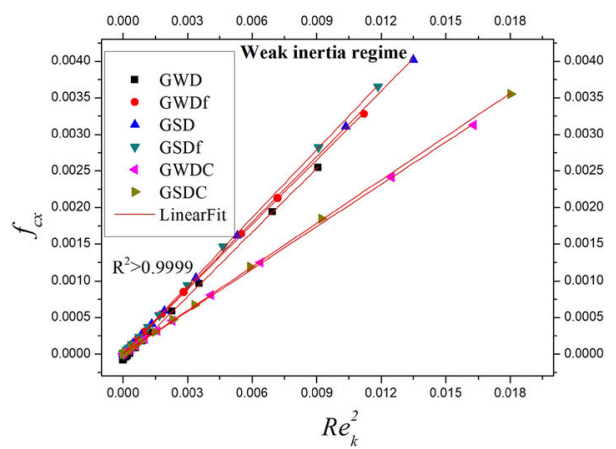

(a) Weak inertia regime

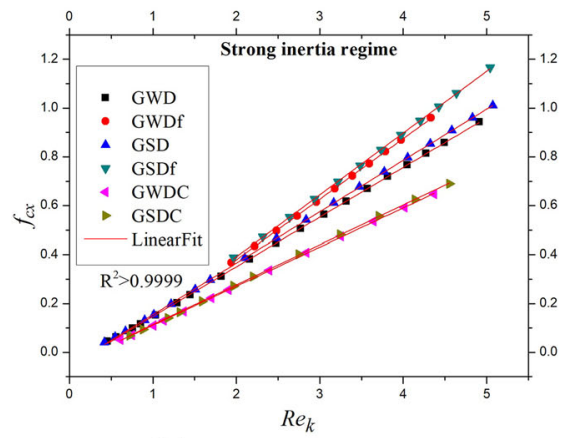

(b) Strong inertia regime

Fig. 7 Variation of $f_{c x}$ versus $R e_{k}^{2}$ (resp. $R e_{k}$ ) for the weak (resp. strong) inertia regime. Data points results from an average over the ten realizations for each structure

Table $3 R e_{k}$ intervals for the different flow regimes and $R e_{k t}$ values

\begin{tabular}{lllll}
\hline Structure & Weak $\left(R e_{k}\right)$ & $R e_{k t}$ & Strong $\left(R e_{k}\right)$ & Beyond $\left(R e_{k}\right)$ \\
\hline GWD & {$[1.19 \mathrm{e}-5,0.1]$} & 0.35 & {$[0.46,6.38]$} & \\
GWDf & {$[1.06 \mathrm{e}-6,0.11]$} & 0.42 & {$[1.94,3.98]$} & {$[3.98,5.59]$} \\
GSD & {$[1.46 \mathrm{e}-5,0.12]$} & 0.35 & {$[0.42,5.56]$} & \\
GSDf & {$[1.37 \mathrm{e}-5,0.11]$} & 0.41 & {$[1.97,4.64]$} & {$[4.64,5.04]$} \\
GWDC & {$[1.6 \mathrm{e}-5,0.13]$} & 0.41 & {$[0.61,4.37]$} & {$[4.37,7.56]$} \\
GSDC & {$[1.92 \mathrm{e}-5,0.13]$} & 0.41 & {$[0.72,4.55]$} & {$[4.55,8.49]$} \\
LWD (Agnaou 2015) & {$[1.51 \mathrm{e}-5,0.22]$} & 0.72 & {$[1.13,5.16]$} & {$[5.54,18.58]$} \\
LWDC (Agnaou 2015) & {$[1.71 \mathrm{e}-5,0.26]$} & 0.83 & {$[1.27,6.77]$} & {$[7.14,19.87]$} \\
OS (Agnaou 2015) & {$[0.03,0.33]$} & 0.75 & {$[0.89,2.31]$} & {$[8.16,23.41]$} \\
OC (Agnaou 2015) & {$[0.03,0.48]$} & 0.97 & {$[1.1,2.68]$} & {$[5.53,21.09]$} \\
\hline
\end{tabular}

porous materials. The weak and strong inertia regimes are separated by a transitional flow characterized again by a Reynolds number interval.

Following a nomenclature used in a previous work (Lasseux et al. 2011), namely

$$
\begin{aligned}
& f_{c x}=A R e_{k}^{2}+B \text { in the weak inertia regime } \\
& f_{c x}=C R e_{k}+D \quad \text { in the strong inertia regime }
\end{aligned}
$$

the above-mentioned linear fits yield the coefficients $A, B, C$ and $D$ in the two regimes as reported in Table 4. From these results, it must be noted that the values of $B$ and $D$ are extremely small and have a negligible contribution. More importantly, it is striking to observe that $A$ and $C$ are significantly larger for GD than for LD structures, equivalent to an increase of the inertia intensity, extending a conclusion reached earlier between LD and ordered structures (Lasseux et al. 2011).

The Reynolds number interval corresponding to the transitional flow between weak and strong inertia can be characterized by a single Reynolds number, $R e_{k t}$, which is the one minimizing the error between the weak and strong inertia correlations in Eqs. (14a) and (14b) hence given by

$$
R e_{k t}=\frac{C}{2 A}
$$


Table 4 Values and standard errors $(\sigma)$ of the coefficients in correlations (14a) and (14b). Note that for LWD (Lasseux et al. 2011) and LSD (Lasseux et al. 2011), coefficients are the average values obtained on the fits performed on 10 different realizations and $\sigma$ is the standard deviation of all the values

\begin{tabular}{lllll}
\hline Structures & $A(\sigma)$ & $B(\sigma)$ & $C(\sigma)$ & $D(\sigma)$ \\
\hline GWD & $0.291(5.3 \mathrm{e}-4)$ & $-8.40 \mathrm{e}-5(1.6 \mathrm{e}-6)$ & $0.203(4.5 \mathrm{e}-4)$ & $-0.06(1.3 \mathrm{e}-3)$ \\
GWDf & $0.294(6.9 \mathrm{e}-4)$ & $4.71 \mathrm{e}-6(2.5 \mathrm{e}-6)$ & $0.248(9.2 \mathrm{e}-4)$ & $-0.12(3 \mathrm{e}-3)$ \\
GSD & $0.299(7.3 \mathrm{e}-4)$ & $6.57 \mathrm{e}-5(3.3 \mathrm{e}-6)$ & $0.210(4.1 \mathrm{e}-4)$ & $-0.05(1.2 \mathrm{e}-3)$ \\
GSDf & $0.309(6.2 \mathrm{e}-4)$ & $4.57 \mathrm{e}-6(2.5 \mathrm{e}-6)$ & $0.253(8.8 \mathrm{e}-4)$ & $-0.12(3.2 \mathrm{e}-3)$ \\
GWDC & $0.193(4.1 \mathrm{e}-4)$ & $4.30 \mathrm{e}-6(2.3 \mathrm{e}-6)$ & $0.160(4.6 \mathrm{e}-4)$ & $-0.05(1.2 \mathrm{e}-3)$ \\
GSDC & $0.198(5.3 \mathrm{e}-4)$ & $5.30 \mathrm{e}-6(2.9 \mathrm{e}-6)$ & $0.163(5 \mathrm{e}-4)$ & $-0.05(1.3 \mathrm{e}-3)$ \\
LWD (Lasseux et al. 2011) & $0.070(6.4 \mathrm{e}-3)$ & $7.10 \mathrm{e}-6(2.1 \mathrm{e}-6)$ & $0.10(8 \mathrm{e}-3)$ & $-4.8 \mathrm{e}-2(5.9 \mathrm{e}-3)$ \\
LWD (Agnaou et al. 2017) & 0.0683 & & 0.0989 & \\
LSD (Lasseux et al. 2011) & $0.20(4.9 \mathrm{e}-2)$ & $6.8 \mathrm{e}-6(1.6 \mathrm{e}-6)$ & $0.15(1.2 \mathrm{e}-2)$ & $-4.1 \mathrm{e}-2(6.6 \mathrm{e}-3)$ \\
LWDC (Agnaou et al. 2017) & 0.0631 & & 0.1042 & $-6.7 \mathrm{e}-3$ \\
OS (Lasseux et al. 2011) & 0.0103 & $1.3 \mathrm{e}-6$ & 0.0153 & 0.0153 \\
OS (Agnaou et al. 2017) & 0.0102 & & 0.0411 & \\
OC (Agnaou et al. 2017) & 0.0212 & & &
\end{tabular}

The values of $R e_{k t}$ for the different structures are listed in Table 3. As can be seen from this table, for both media made up of circular and square inclusions, $R e_{k t}$ decreases as disorder is introduced from ordered to LD and, further, to GD structures, without any impact of weak or strong disorder in the latter case. Values of $R e_{k t}$ for GSDf and GWDf structures are intermediate between those for GWD (which is the same as for GSD) and LWD media. Moreover, $R e_{k t}$ is always larger for the structure made up of circular inclusions compared to the corresponding one including square obstacles.

Beyond the strong inertia regime, the dependency of $f_{c x}$ with respect to $R e_{k}$ can be fitted according to the following polynomial correlation (Agnaou (2015); Lasseux et al. (2011)):

$$
f_{c x}=E R e_{k}^{2}+F R e_{k}+G
$$

and an illustration of the corresponding coefficients is given in Table 5 for GWDf, GWDC and GSDC together with some recalls for ordered and LD structures reported in Agnaou et al. (2017). As can be seen from Table 3, the range of Reynolds numbers where this regime is observed on GD structures is significantly reduced compared to ordered and LD structures. In particular, this regime was not identified for $R e_{k} \leq R e_{k c}$ for GWD and GSD structures, which means the bifurcation just follows strong inertia in these two pore geometries. Moreover, it must be noted that the values of $E$ remain quite small compared to $F$ and $G$. Because the maximum value of $R e_{k}$ where the correlation in Eq. (16) applies is about 8.5, the contribution of the quadratic term in this correlation remains quite small with respect to the linear term, suggesting that a linear correlation, close to that in the strong inertia regime, is robust in the Reynolds number range beyond the strong inertia regime. Indeed, with a less restrictive constraint on the Pearson coefficient to fit the data, regime beyond strong inertia identified above would be merged into the strong inertia regime. This remark does not apply to the LWD or ordered structures for which the maximum Reynolds number in the regime beyond strong inertia is about 20 or more. These observations may be better highlighted while comparing the $R e_{k}$ dependence of $\frac{\partial f_{c x}}{\partial R e_{k}}$, normalized by the corresponding maximum value, over the whole steady regime for the different structures under consideration. This is represented in Fig. 8, 
Table 5 Coefficients of the polynomial fit for the regime beyond strong inertia

\begin{tabular}{llll}
\hline Structure & $E(\sigma)$ & $F(\sigma)$ & $G(\sigma)$ \\
\hline GWDf & $3.8 \mathrm{e}-3(1.86 \mathrm{e}-5)$ & $0.226(1.85 \mathrm{e}-4)$ & $-0.080(4.55 \mathrm{e}-4)$ \\
GWDC & $1.8 \mathrm{e}-3(2.84 \mathrm{e}-5)$ & $0.168(3.50 \mathrm{e}-4)$ & $-0.052(1.06 \mathrm{e}-3)$ \\
GSDC & $1.8 \mathrm{e}-3(3.60 \mathrm{e}-5)$ & $0.170(4.84 \mathrm{e}-4)$ & $-0.045(1.59 \mathrm{e}-3)$ \\
LWD (Agnaou et al. 2017) & $-8.3 \mathrm{e}-4$ & 0.0915 & \\
LWDC (Agnaou et al. 2017) & $-1.17 \mathrm{e}-3$ & 0.1025 & \\
OS (Agnaou et al. 2017) & $-1 \mathrm{e}-4$ & 0.0053 & \\
OC (Agnaou et al. 2017) & $-4.6 \mathrm{e}-4$ & 0.0228 & \\
\hline
\end{tabular}

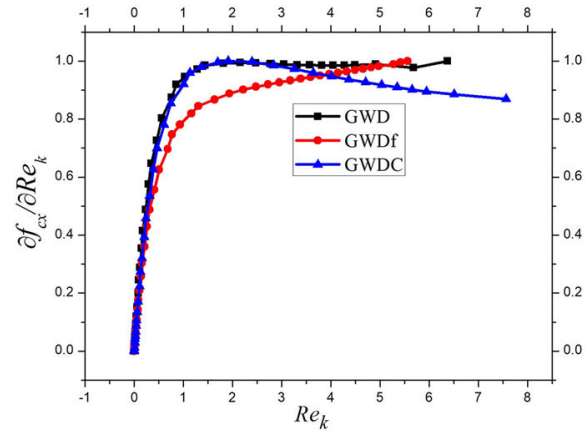

(a) GD structures with cylinders of uniform size

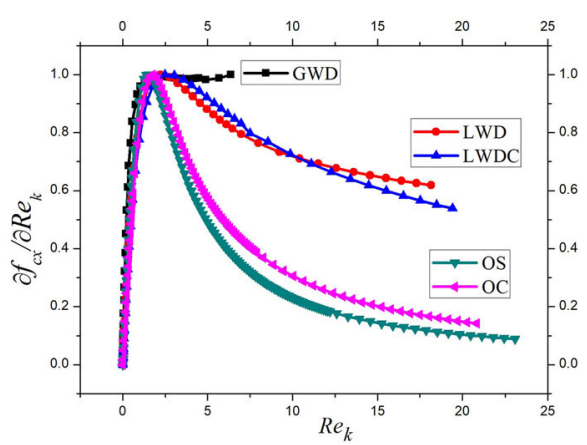

(b) Ordered and locally disordered structures

Fig. 8 Variation of $\frac{\partial f_{c x}}{\partial R e_{k}}$, normalized by the corresponding maximum value, with $R e_{k}$ in the steady flow regime for GD structures with cylinders of uniform size (a) and comparison with ordered and locally disordered structures (b)

except for the GSD structures (see the justification below). For all structures, at low Reynolds numbers, a linear relationship is obtained in this graph, as expected in the weak inertia regime. The quadratic correction to Darcy's law in the strong inertia regime at larger values of $R e_{k}$, corresponding to a classical Forchheimer correction widely used in the literature, is much more robust for GD (in particular for GWD and GWDC) (Fig. 8a) than for ordered or LD structures (see Fig 8b). As mentioned above, for the GWDf structure, no significant change with respect to the strong inertia regime is observed in the regime identified beyond strong inertia, confirming that a quadratic correction remains quite relevant in this Reynolds number range $\left(3.98 \leq R e_{k} \leq 5.59\right)$.

As final important remarks, it should be noted that:

- For ordered structures, inertia intensity is larger for media with circular inclusions (OC) than for those with square obstacles (OS)

- Inertia is more intense for GD (either weakly or strongly disordered) structures made up of square obstacles than for those with circular inclusions. This might be physically explained by the fact that, since the wake downstream of a square cylinder (see illustrations in Agnaou et al. (2016)) is much wider than behind a circular cylinder (Yuce and Kareem 2016; Zaki et al. 1994), interaction of the wakes in structures with squares become stronger than that with circular cylinders, thus increasing inertia intensity. This 
effect is not present in ordered structures as wakes are well separated in the direction orthogonal to the flow.

- As can be seen from Table 4 and Figs. 6 and 7, inertia is not significantly altered by polydispersity for globally disordered media, i.e., GS or GW structures feature almost the same behavior. This contrasts with LD structures as previously observed in Lasseux et al. (2011). This might be attributed to the fact that global disorder prevails over size distribution of the inclusions. From that, it may be concluded that one-phase flow analysis can be restricted to monodisperse GD structures. This justifies why attention was only focused on these types of media in Fig. 8.

- Random inclinations of square obstacles leads to slightly larger inertial effects in GD structures. A similar phenomenon was observed for ordered structures (OS and OC) in the work of Agnaou et al. (2017), in which an average pressure gradient oriented at an angle $\theta=45^{\circ}$ with respect to the lattice vectors of the geometrical unit cell yields more intense inertia compared to the case where $\theta=0^{\circ}$. This phenomenon needs to be further investigated in random structures as flow topologies differ a lot between ordered and GD structures.

\section{Conclusions}

Effect of the microscopic structure on single-phase incompressible flow in 2D model porous media has been thoroughly investigated in this paper, widely extending previous analyses on this subject. Firstly, six types of spatially periodic porous structures were generated to introduce more structural disorder, compared to the ordered and locally disordered media studied so far in the literature (Lasseux et al. 2011; Agnaou et al. 2017). The critical Reynolds number at which laminar flow becomes unsteady was determined. On the basis of this critical value, numerical simulations of steady laminar flow were performed in order to thoroughly investigate the inertial correction to Darcy's law.

It was found that inertial flow is significantly affected by the microstructure. The critical Reynolds number based on the obstacle size becomes much smaller as more disorder is introduced into the pore structures. In contrast with locally disordered structures considered in previous works, the critical Reynolds number for globally disordered structures significantly varies from one realization to another, suggesting that, for the size of the unit cell employed in this work, an ensemble average is required to capture a value representative of the same structure of infinite extent. The critical Reynolds number is significantly affected by the cylinder shape, but hardly depends on the size distribution and inclination of the cylinders.

The Reynolds number interval corresponding to the weak inertia regime shrinks when pore structures become more disordered. Compared to the weak inertia regime, the strong inertia regime is much more robust for GD structures than for less disordered structures studied in previous works. The intensity of inertia increases with structural disorder and is mainly influenced by the shape of the obstacles but is slightly affected by the inclination of the square cylinders and is barely disturbed by the size distribution of the obstacles.

While the present analysis has been dedicated to the homogeneous part of a porous medium, a lot remains to be done to investigate inertial effects close to the macroscopic boundaries of the system. A derivation of a macroscopic model valid everywhere, including in the neighborhood of the macroscopic boundaries, was proposed in Paéz-García et al. (2017). However, specific numerical simulations must be performed to determine the modifications of the inertial correction expected close to these boundaries. 
Acknowledgements The financial support from the program of China Scholarship Council (No. 201506830049) and computational resources provided by the computing facility MCIA (Mésocentre de Calcul Intensif Aquitain) of the University of Bordeaux are gratefully acknowledged.

\section{Appendix}

In this appendix, the mesh convergence analysis is summarized. It was performed by evaluating the impact of the mesh size on $k^{*}=\frac{\overline{k_{x x}^{*}}+\overline{k_{y y}^{*}}}{2}, \overline{k_{x x}^{*}}$ and $\overline{k_{y y}^{*}}$ being the dimensionless diagonal permeability components averaged over the ten realizations, the off-diagonal components, $k_{x y}^{*}$, remaining negligible (see Sect. 3.2.1). The variation of $k^{*}$ versus the number of grid blocks is reported in Fig. 9. It must be noted that the standard deviation on diagonal term of the permeability is of the order of $10^{-4}$, a value which is roughly two orders of magnitude smaller than the typical value of $k^{*}$, showing that each of the ten realizations provides a quite accurate value of the permeability (see Sect. 3.2.1 for additional comments).

Convergence was assessed by considering a relative variation of $k^{*}$ smaller than $0.5 \%$, taking the reference value as the one obtained for the finest mesh size (i.e., $\sim 3.2 \times 10^{6}$ grid blocks). From this criterion, a mesh made up of around $2.2 \times 10^{6}$ grid blocks was found adequate for all the structures. To make sure this convergence remains for larger Reynolds numbers, the relative error on the $x$-component of $\mathbf{f}_{c}, f_{c x}$, for these two grid block numbers was computed on a realization of a GWD structure for the maximum value of the Reynolds number considered for this structure (see Sect. 3.1), namely $R e^{*}=18,000$. The relative error was found to be around $0.22 \%$, confirming the choice for the mesh size.

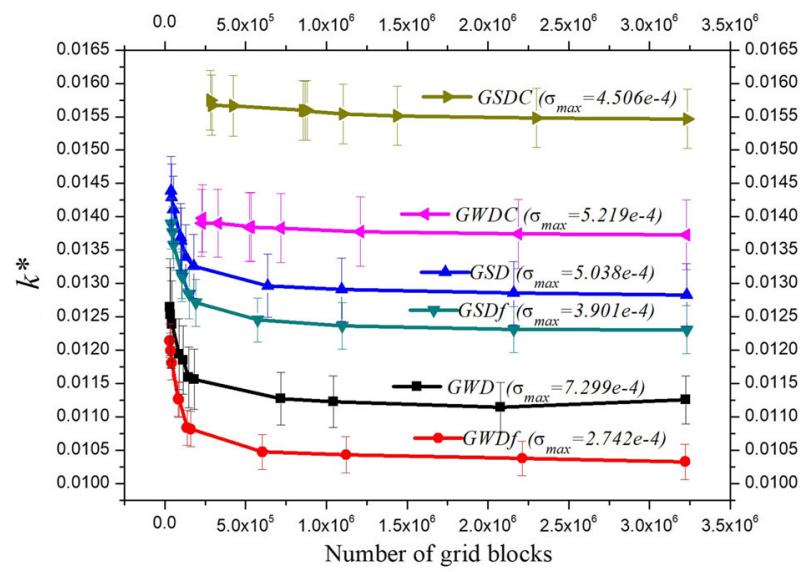

Fig. 9 Variation of the dimensionless average permeability, $k^{*}$, with the grid block number. $\sigma_{\max }$ is the maximum of the standard deviation, $\sigma$, of $k^{*}$ obtained for the different number of grid blocks. Error bars represent the $\pm \sigma$ permeability span 


\section{References}

Abbasian Arani, A.A.: Sur quelques aspects des écoulements inertiels mono- et diphasique en milieu poreux. $\mathrm{PhD}$ thesis, Université de Bordeaux I (2006)

Agnaou, M.: Une étude numérique des écoulements mono et diphasique inertiels en milieux poreux. $\mathrm{PhD}$ thesis, Arts et Métiers ParisTech, Centre de Bordeaux, Talence (2015)

Agnaou, M., Lasseux, D., Ahmadi, A.: From steady to unsteady laminar flow in model porous structures: an investigation of the first Hopf bifurcation. Comput. Fluids 136, 67-82 (2016). https://doi.org/10.1016/j. compfluid.2016.05.030

Agnaou, M., Lasseux, D., Ahmadi, A.: Origin of the inertial deviation from Darcy's law: an investigation from a microscopic flow analysis on two-dimensional model structures. Phys. Rev. E 96, 043,105 (2017). https://doi.org/10.1103/PhysRevE.96.043105

Amaral Souto, H.P., Moyne, C.: Dispersion in two-dimensional periodic porous media. Part I. hydrodynamics. Phys. Fluids 9(8), 2243-2252 (1997). https://doi.org/10.1063/1.869365

Amestoy, P.R., Duff, I.S., L'Excellent, J., Koster, J.: A fully asynchronous multifrontal solver using distributed dynamic scheduling. SIAM J. Matrix Anal. Appl. 23(1), 15-41 (2001). https://doi.org/10.1137/ S0895479899358194

Andrade, J.S., Costa, U.M.S., Almeida, M.P., Makse, H.A., Stanley, H.E.: Inertial effects on fluid flow through disordered porous media. Phys. Rev. Lett. 82, 5249-5252 (1999). https://doi.org/10.1103/PhysRevLett. 82.5249

Auriault, J.L., Geindreau, C., Orgéas, L.: Upscaling Forchheimer law. Transp. Porous Media 70(2), 213-229 (2007). https://doi.org/10.1007/s11242-006-9096-x

Barrère, J.: Modélisation des écoulements de Stokes et Navier-Stokes en milieu poreux. PhD thesis, Université de Bordeaux I (1990)

Chai, Z.H., Shi, B.C., Lu, J.H., Guo, Z.L.: Non-Darcy flow in disordered porous media: a lattice-Boltzmann study. Comput. Fluids 39(10), 2069-2077 (2010). https://doi.org/10.1016/j.compfluid.2010.07.012

Chen, J.H., Pritchard, W.G., Tavener, S.J.: Bifurcation for flow past a cylinder between parallel planes. J. Fluid Mech. 284, 23-41 (1995). https://doi.org/10.1017/S0022112095000255

Clavier, R.: Etude expérimentale et modélisation des pertes de pression lors du renoyage d'un lit de débris. $\mathrm{PhD}$ thesis, Université de Toulouse (2015)

COMSOL Multiphysics: Comsol multiphysics user's guide version 4.3a (2012)

Edwards, D.A., Shapiro, M., Bar-Yoseph, P., Shapira, M.: The influence of Reynolds number upon the apparent permeability of spatially periodic arrays of cylinders. Phys. Fluids A 2(1), 45-55 (1990). https://doi.org/ $10.1063 / 1.857691$

Enayati, H., Braun, M., Chandy, A.: Numerical simulations of porous medium with different permeabilities and positions in a laterally-heated cylindrical enclosure for crystal growth. J. Cryst. Growth 483, 65-80 (2018). https://doi.org/10.1016/j.jcrysgro.2017.11.019

Forchheimer, P.: Wasserbewgung durch Boden. Vereines Deutscher Ingenieure XXXXV(45), 1782-1788 (1901)

Fourar, M., Radilla, G., Lenormand, R., Moyne, C.: On the non-linear behavior of a laminar single-phase flow through two and three-dimensional porous media. Adv. Water Resour. 27(6), 669-677 (2004). https:// doi.org/10.1016/j.advwatres.2004.02.021

Franke, R., Rodi, W., Schnung, B.: Numerical calculation of laminar vortex-shedding flow past cylinders. J. Wind Eng. Ind. Aerodyn. 35, 237-257 (1990). https://doi.org/10.1016/0167-6105(90)90219-3

Gera, B., Sharma, P.K., Singh, R.K.: CFD analysis of 2D unsteady flow around a square cylinder. Int. J. Appl. Eng. Res. 1(3), 602 (2010)

Gray, W.G.: A derivation of the equations for multi-phase transport. Chem. Eng. Sci. 30(2), 229-233 (1975). https://doi.org/10.1016/0009-2509(75)80010-8

Hill, R.J., Koch, D.L.: Moderate-Reynolds-number flow in a wall-bounded porous medium. J. Fluid Mech. 453, 315-344 (2002). https://doi.org/10.1017/S002211200100684X

Jackson, C.P.: A finite-element study of the onset of vortex shedding in flow past variously shaped bodies. J. Fluid Mech. 182, 23-45 (1987). https://doi.org/10.1017/S0022112087002234

Kawaguti, M.: The critical Reynolds number for the flow past a sphere. J. Phys. Soc. Jpn. 10, 694-699 (1955). https://doi.org/10.1143/JPSJ.10.694

Kelkar, K.M., Patankar, S.V.: Numerical prediction of vortex shedding behind a square cylinder. Int. J. Numer. Methods Fluids 14(3), 327-341 (1992). https://doi.org/10.1002/fld.1650140306

Koch, D.L., Ladd, A.J.C.: Moderate Reynolds number flows through periodic and random arrays of aligned cylinders. J. Fluid Mech. 349, 31-66 (1997). https://doi.org/10.1017/S002211209700671X

Kumar, B., Mittal, S.: Prediction of the critical Reynolds number for flow past a circular cylinder. Comput. Methods Appl. Mech. Eng. 195(44-47), 6046-6058 (2006). https://doi.org/10.1016/j.cma.2005.10.009 
Kumar, S.R., Sharma, A., Agrawal, A.: Simulation of flow around a row of square cylinders. J. Fluid Mech. 606, 369-397 (2008). https://doi.org/10.1017/S0022112008001924

Lankadasu, A., Vengadesan, S.: Onset of vortex shedding in planar shear flow past a square cylinder. Int. J. Heat Fluid Flow 29(4), 1054-1059 (2008). https://doi.org/10.1016/j.ijheatfluidflow.2008.02.016

Lasseux, D., Abbasian Arani, A.A., Ahmadi, A.: On the stationary macroscopic inertial effects for one phase flow in ordered and disordered porous media. Phys. Fluids 23(7), 073103 (2011). https://doi.org/10. $1063 / 1.3615514$

Lasseux, D., Valdés-Parada, F.J.: On the developments of Darcy's law to include inertial and slip effects. A century of fluid mechanics: 1870-1970. C. R. Méc. 345(9), 660-669 (2017). https://doi.org/10.1016/j. crme.2017.06.005

Lee, S., Yang, J.: Modeling of Darcy-Forchheimer drag for fluid flow across a bank of circular cylinders. Int. J. Heat Mass Transf. 40(13), 3149-3155 (1997). https://doi.org/10.1016/s0017-9310(96)00347-x

Liu, M., Chen, Y., Zhan, H., Hu, R., Zhou, C.: A generalized forchheimer radial flow model for constant-rate tests. Adv. Water Resour. 107, 317-325 (2017). https://doi.org/10.1016/j.advwatres.2017.07.004

Marsden, J.E., McCracken, M.: The Hopf Bifurcation and Its Applications. Applied Mathematical Sciences, vol. 19. Springer, New York (1976)

Mei, C.C., Auriault, J.L.: The effect of weak inertia on flow through a porous medium. J. Fluid Mech. 222, 647-663 (1991). https://doi.org/10.1017/S0022112091001258

Mizushima, J., Akinaga, T.: Vortex shedding from a row of square bars. Fluid Dyn. Res. 32(4), 179-191 (2003). https://doi.org/10.1016/S0169-5983(03)00016-9

Paéz-García, C.T., Valdés-Parada, E.J., Lasseux, D.: Macroscopic momentum and mechanical energy equations for incompressible single-phase flow in porous media. Phys. Rev. E 95(2), 023101 (2017)

Payri, F., Broatch, A., Serrano, J., Piqueras, P.: Experimental-theoretical methodology for determination of inertial pressure drop distribution and pore structure properties in wall-flow diesel particulate filters (dpfs). Energy 36(12), 6731-6744 (2011). https://doi.org/10.1016/j.energy.2011.10.033

Skjetne, E., Auriault, J.L.: New insights on steady, non-linear flow in porous media. Eur. J. Mech. B/Fluids 18(1), 131-145 (1999). https://doi.org/10.1016/S0997-7546(99)80010-7

Sohankar, A., Norberg, C., Davidson, L.: Low-Reynolds-number flow around a square cylinder at incidence: study of blockage, onset of vortex shedding and outlet boundary condition. Int. J. Numer. Methods Fluids 26(1), 39-56 (1998). https://doi.org/10.1002/(SICI)1097-0363(19980115)26:1<39::AIDFLD623>3.0.CO;2-P

Soulaine, C., Quintard, M.: On the use of a Darcy-Forchheimer like model for a macro-scale description of turbulence in porous media and its application to structured packings. Int. J. Heat Mass Transf. 74, 88-100 (2014). https://doi.org/10.1016/j.ijheatmasstransfer.2014.02.069

Valdés-Parada, F.J., Lasseux, D., Bellet, F.: A new formulation of the dispersion tensor in homogeneous porous media. Adv. Water Resour. 90, 70-82 (2016). https://doi.org/10.1016/j.advwatres.2016.02.012

Weller, H.G., Tabor, G., Jasak, H., Fureby, C.: A tensorial approach to computational continuum mechanics using object-oriented techniques. Comput. Phys. 12(6), 620-631 (1998). https://doi.org/10.1063/1. 168744

Whitaker, S.: Advances in theory of fluid motion in porous media. Ind. Eng. Chem. 61(12), 14-28 (1969). https://doi.org/10.1021/ie50720a004

Whitaker, S.: The Forchheimer equation: a theoretical development. Transp. Porous Media 25(1), 27-61 (1996). https://doi.org/10.1007/BF00141261

Whitaker, S.: The Method of Volume Averaging: Theory and Applications of Transport in Porous Media. Kluwer Academic, Dordrecht (1999). https://doi.org/10.1007/978-94-017-3389-2

Yazdchi, K., Srivastava, S., Luding, S.: On the transition from creeping to inertial flow in arrays of cylinders. Mech. Solids Struct. Fluids 9, 767-772 (2010)

Yuce, M.I., Kareem, D.A.: A numerical analysis of fluid flow around circular and square cylinders. J. Am. Water Works Assoc. 108(10), 546-554 (2016). https://doi.org/10.5942/jawwa.2016.108.0141

Zaki, T.G., Sen, M., Gad-El-Hak, M.: Numerical and experimental investigation of flow past a freely rotatable square cylinder. J. Fluids Struct. 8(7), 555-582 (1994). https://doi.org/10.1016/S0889-9746(94)900205 